\title{
Informational features of Fermionic systems
}

\author{
Alessandro Tosini* \\ Dipartimento di Fisica dell'Università degli Studi di Pavia \\ E-mail: alessandro.tosini@unipv.it
}

The anticommutation of Fermionic fields raises the problem of simulating the evolution of Fermionic systems by means of commuting quantum systems, say qubits. We tackle [1,2] the issue of retaining locality of Fermionic operations considering local Fermionic modes as the elementary systems of an operational probabilistic theory. The locality of Fermionic operations, namely operations on systems that are not causally connected must commute, implies the parity superselection rule that inhibits the superposition of states with an even and an odd number of excitations. As a result the Fermionic theory lacks two distinctive traits of quantum theory, the local tomography and the monogamy of the entanglement.

References

[1] D'Ariano, Giacomo Mauro, Franco Manessi, Paolo Perinotti, and Alessandro Tosini, The Feynman problem and Fermionic entanglement: Fermionic theory versus qubit theory, accepted on Int. J. Mod. Phys. A, arXiv preprint arXiv:1403.2674 (2014).

[2] D'Ariano, Giacomo Mauro, Franco Manessi, Paolo Perinotti, and Alessandro Tosini, Fermionic computation is non-local tomographic and violates monogamy of entanglement, arXiv preprint arXiv:1307.7902 (2013).

Frontiers of Fundamental Physics 14 - FFP14,

15-18 July 2014

Aix Marseille University (AMU) Saint-Charles Campus, Marseille

\footnotetext{
*Speaker.
} 